\title{
dspace.vutbr.cz
}

\section{An Empirical Study of Fatigue-Induced Electrical Impedance Models of Biceps Tissues}

\author{
HERENCSÁR, N.
}

Proceedings of the 2020 12th International Congress on Ultra Modern Telecommunications and Control Systems and Workshops (ICUMT), pp. 58-61

elSBN: 978-1-7281-9281-9

ISSN: 2157-023X

DOl: https://doi.org/10.1109/ICUMT51630.2020.9222426

Accepted manuscript

(C2020 IEEE. Personal use of this material is permitted. Permission from IEEE must be obtained for all other uses, in any current or future media, including reprinting/republishing this material for advertising or promotional purposes, creating new collective works, for resale or redistribution to servers or lists, or reuse of any copyrighted component of this work in other works. HERENCSÁR, N., "An Empirical Study of Fatigue-Induced Electrical Impedance Models of Biceps Tissues", 12th International Congress on Ultra Modern Telecommunications and Control Systems and Workshops (ICUMT), 2020. DOI: 10.1109/ICUMT51630.2020.9222426. Final version is available at https://ieeexplore.ieee.org/document/9222426 


\title{
An Empirical Study of Fatigue-Induced Electrical Impedance Models of Biceps Tissues
}

\author{
Norbert Herencsar \\ Department of Telecommunications, Brno University of Technology \\ Brno, Czech Republic \\ herencsn@feec.vutbr.cz
}

\begin{abstract}
Electrical impedance myography (EIM) is a noninvasive approach to muscle assessment based on the measurement of the electrical impedance in frequency range of interest. In this paper, fatigue-induced electrical impedance models of biceps tissues are investigated. After the dataset used is briefly described, complete set of parameters of the modified Fricke model, utilizing Foster I RC network-based fractional-order capacitor (FOC), are computed. The goodness of fitting of proposed FOCs and bioimpedance models were evaluated visually and statistically. The fit accuracy of designed electrical impedance models is $\mathbf{R}_{\mathrm{R}, \mathrm{X}}^{2} \geq 0.9978$. Proposed models provide more intuitive representation of the electrical behavior of biceps tissues. EIA standard compliant E96 series lumped parameter-based practical models give an appropriate explanation of fatigue effect on biceps tissues from resistance and capacitance point of view.

Index Terms-biceps tissue, bioimpedance, Cole model, constant phase element, CPE, electrical impedance myography, EIM, Foster I network, fractional calculus, modified Fricke model
\end{abstract}

\section{INTRODUCTION}

Electrical impedance myography (EIM) is a form of neuromuscular evaluation in which alternating current at frequency of interest is injected into the body via two current electrodes and the resulted voltage pattern over a selected muscle is measured using voltage electrodes [1]-[5]. Figure 1(a) illustrates the current flow at low and high frequencies in biological tissues. One of the first electrical impedance models for the biological tissues so-called bioimpedance was introduced by Fricke [6]. In the modified Fricke model depicted in Fig. 1(b) the capacitive element of the Fricke model is replaced by a fractional-order capacitor (FOC) or so-called capacitive constant phase element (CPE), whose impedance is $Z_{\mathrm{CPE}}=1 / s^{\alpha} C$ in the $s$-domain, where $C$ denotes pseudo-capacitance (with unit Farad $\cdot \sec ^{\alpha-1}$ or $\mathrm{F} \cdot \sec ^{\alpha-1}$ ) and $\alpha$ is its order in range $0<\alpha<1$ [7]. The impedance of FOC has frequency dependent real part and its magnitude varies by $-20 \alpha \mathrm{dB}$ per decade of frequency, while the phase is constant in full frequency range and equal to $-\alpha \pi / 2$. The modified Fricke model is the parallel version of well-known Cole model [8], [9], however it provides a better intuitive representation of the electrical behavior of biological tissues. It is composed of an extra-cellular fluid resistance $\left(R_{\mathrm{ECF}}\right)$ placed in parallel with a resistive element $\left(R_{\mathrm{ICF}}\right)$ in serial with a FOC $\left(C_{\mathrm{M} \alpha}\right)$. The element

The research results described in this paper are supported by The Czech Science Foundation, project No. 19-24585S.

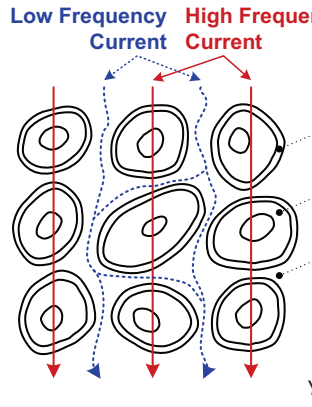

(a)

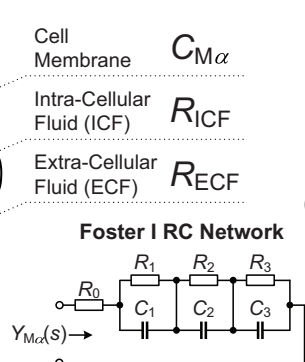

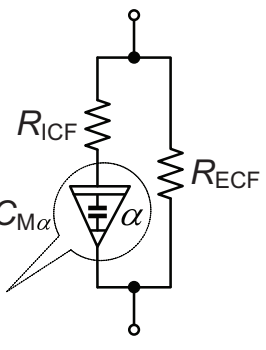

(b)
Fig. 1. (a) Demonstration of current flow in a biological tissue and (b) modified Fricke circuit model using CPE.

$R_{\mathrm{ICF}}$ directly represents intra-cellular fluid resistance, while the $C_{\mathrm{M} \alpha}$ corresponds to the capacitance of cells created by their insulating cell membranes [10]. Its impedance in the $s$-domain can be expressed as $Z(s)$ Modified-Fricke $=$ $R_{\mathrm{ECF}}\left(R_{\mathrm{ICF}} C_{\mathrm{M} \alpha} s^{\alpha}+1\right) /\left[1+\left(R_{\mathrm{ECF}}+R_{\mathrm{ICF}}\right) s^{\alpha} C_{\mathrm{M} \alpha}\right]$, where $s=j \omega$. The resistances at zero and infinite frequencies are $R_{\mathrm{ECF}}$ and $R_{\mathrm{ECF}} \| R_{\mathrm{ICF}}$, respectively.

Muscle fatigue, as a common physiological phenomenon, has attracted much attention in the recent past [3]-[5]. Therefore, this study aims to develop practical fatigue-induced electrical impedance models of the biceps tissues (musculus biceps brachii L.).

\section{Materials And Methods}

This Section briefly describes the initial dataset [3] used in this paper.

\section{A. Description of Participants and Applied Exercises}

In [3], the electrical impedance measurements were collected from the left and right biceps of 18 participants with an average age of $22.2 \pm 3.2$ years immediately prior to and immediately after completing an exercise protocol to fatigue the biceps brachii muscles. Each participant executed a fatiguing exercise protocol using dumbbell biceps curls at either $60 \%$ (10 participants) or $75 \%$ (8 participants) of their previously assessed one-repetition maximum (1-RM) until task failure. The 1-RM value for each participant is the maximum weight that they were able to successfully lift for the dumbbell biceps curl exercise. Further details regarding the grouping of participants and the description of protocol are given in [3]. 
TABLE I

Equivalent Circuit Parameters of Pre- and Post-Fatigue Impedance Models From the Left and Right Biceps Tissues.

\begin{tabular}{|c|c|c|c|c|c|c|c|c|c|c|c|c|c|c|c|c|c|}
\hline \multirow{9}{*}{ 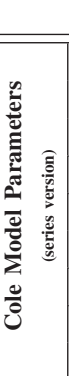 } & & \multicolumn{8}{|c|}{ Left Biceps } & \multicolumn{8}{|c|}{ Right Biceps } \\
\hline & \multirow[t]{2}{*}{ Elements } & \multicolumn{2}{|c|}{$\begin{array}{l}R_{\infty} \\
(\Omega)\end{array}$} & \multicolumn{2}{|c|}{$\begin{array}{l}R_{1} \\
(\Omega)\end{array}$} & \multicolumn{2}{|c|}{$\begin{array}{c}C_{\alpha} \\
\left(\mu \mathrm{F} \cdot \sec ^{\alpha-1}\right)\end{array}$} & \multicolumn{2}{|c|}{$\begin{array}{c}\alpha \\
(-)\end{array}$} & \multicolumn{2}{|c|}{$\begin{array}{l}R_{\infty} \\
(\Omega)\end{array}$} & \multicolumn{2}{|c|}{$\begin{array}{l}R_{1} \\
(\Omega)\end{array}$} & \multicolumn{2}{|c|}{$\begin{array}{c}C_{\alpha} \\
\left(\mu \mathrm{F} \cdot \sec ^{\alpha-1}\right)\end{array}$} & \multicolumn{2}{|c|}{$\begin{array}{c}\alpha \\
(-)\end{array}$} \\
\hline & & Pre & Post & Pre & Post & Pre & Post & Pre & Post & Pre & Post & Pre & Post & Pre & Post & Pre & Post \\
\hline & \multicolumn{17}{|c|}{$60 \%$ 1-RM Group } \\
\hline & Mean & 18.78 & 17.63 & 32.47 & 27.95 & 3.28 & 3.08 & 0.732 & 0.737 & 17.49 & 16.70 & 31.21 & 27.15 & 4.28 & 3.94 & 0.724 & 0.729 \\
\hline & $\pm \mathrm{SD}$ & 5.60 & 6.16 & 5.02 & 4.98 & 1.57 & 2.12 & 0.039 & 0.041 & 7.02 & 7.14 & 5.75 & 4.76 & 3.15 & 2.62 & 0.059 & 0.054 \\
\hline & \multicolumn{17}{|c|}{$75 \%$ 1-RM Group } \\
\hline & Mean & 19.40 & 18.46 & 32.04 & 27.83 & 7.71 & 7.08 & 0.678 & 0.691 & 21.28 & 19.88 & 31.55 & 28.08 & 4.77 & 5.02 & 0.706 & 0.710 \\
\hline & $\pm \mathrm{SD}$ & 6.72 & 7.04 & 8.63 & 7.42 & 4.81 & 4.84 & 0.075 & 0.084 & 7.96 & 8.31 & 6.36 & 6.08 & 2.08 & 2.68 & 0.057 & 0.065 \\
\hline \multirow{8}{*}{ 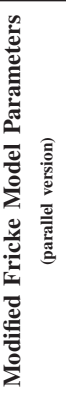 } & \multirow[t]{2}{*}{ Elements } & \multicolumn{2}{|c|}{$\begin{array}{c}R_{\text {ECF }} \\
(\Omega)\end{array}$} & \multicolumn{2}{|c|}{$\begin{array}{c}R_{\mathrm{ICF}} \\
(\Omega)\end{array}$} & \multicolumn{2}{|c|}{$\begin{array}{c}C_{\mathrm{M} \alpha} \\
\left(\mu \mathrm{F} \cdot \sec ^{\alpha-1}\right)\end{array}$} & \multicolumn{2}{|c|}{$\begin{array}{c}\alpha \\
(-)\end{array}$} & \multicolumn{2}{|c|}{$\begin{array}{c}R_{\mathrm{ECF}} \\
(\Omega)\end{array}$} & \multicolumn{2}{|c|}{$\begin{array}{c}R_{\mathrm{ICF}} \\
(\Omega)\end{array}$} & \multicolumn{2}{|c|}{$\begin{array}{c}C_{\mathrm{M} \alpha} \\
\left(\mu \mathrm{F} \cdot \sec ^{\alpha-1}\right)\end{array}$} & \multicolumn{2}{|c|}{$\begin{array}{c}\alpha \\
(-)\end{array}$} \\
\hline & & Pre & Post & Pre & Post & Pre & Post & Pre & Post & Pre & Post & Pre & Post & Pre & Post & Pre & Post \\
\hline & \multicolumn{17}{|c|}{ 60\% 1-RM Group } \\
\hline & $\begin{array}{c}\text { Mean } \\
(\mathrm{R}-\mathrm{E} 96)\end{array}$ & $\begin{array}{c}51.25 \\
(48.7+2.55)\end{array}$ & $\begin{array}{c}45.58 \\
(422|| 51.1)\end{array}$ & $\begin{array}{c}29.64 \\
(26.7+2.94)\end{array}$ & $\begin{array}{c}28.75 \\
(20.5+8.25)\end{array}$ & 1.32 & 1.16 & 0.732 & & $\begin{array}{l}48.70 \\
(48.70)\end{array}$ & $\begin{array}{c}43.85 \\
(40.2+3.65)\end{array}$ & $\begin{array}{c}27.29 \\
(18.2+9.09)\end{array}$ & $\begin{array}{c}26.97 \\
(19.1+7.87)\end{array}$ & 1.76 & 1.51 & 0.724 & 0.729 \\
\hline & $\pm \mathrm{SD}$ & 10.62 & 11.14 & 11.85 & 13.80 & 0.350 & 0.424 & 0.039 & 0.041 & 12.78 & 11.90 & 15.59 & 17.87 & 0.639 & 0.419 & 0.059 & 0.054 \\
\hline & \multicolumn{17}{|c|}{ 75\% 1-RM Group } \\
\hline & $\begin{array}{c}\text { Mean } \\
(\mathrm{R}-\mathrm{E} 96)\end{array}$ & $\begin{array}{c}51.44 \\
(48.7+2.74)\end{array}$ & $\begin{array}{c}46.29 \\
(43.2+3.09)\end{array}$ & $\begin{array}{c}31.15 \\
(30.1+1.05)\end{array}$ & $\begin{array}{c}30.70 \\
(17.4+13.3)\end{array}$ & 2.99 & 2.56 & 0.678 & 0.691 & $\begin{array}{c}52.83 \\
(1.4 \mathrm{k}|| 54.9)\end{array}$ & $\begin{array}{c}47.96 \\
(42.2+5.76)\end{array}$ & $\begin{array}{c}35.63 \\
(26.1+9.53)\end{array}$ & $\begin{array}{c}33.95 \\
(25.5+8.45)\end{array}$ & 1.70 & 1.72 & 0.706 & 0.710 \\
\hline & $\pm \mathrm{SD}$ & 15.35 & 14.45 & 11.95 & 13.71 & 1.52 & 1.28 & 0.075 & 0.084 & 14.32 & 14.39 & 17.91 & 19.68 & 0.410 & 0.478 & 0.057 & 0.065 \\
\hline
\end{tabular}

\section{B. CPE Models via Foster I RC Network}

\section{B. Data Collection and Modeling}

Two current injection electrodes were placed on the lateralis side of the biceps $14 \mathrm{~cm}$ apart with two voltage sensing electrodes each placed $4.67 \mathrm{~cm}$ apart [3]. The impedance measures were collected in frequency range from $10 \mathrm{kHz}$ to $100 \mathrm{kHz}$ using a Keysight E4990A impedance analyzer with a custom-interface to adapt the BNC connectors of the E4990A to a cable-set with the snap connectors for commercial off-theshelf $\mathrm{Ag} / \mathrm{AgCl}$ electrodes. Subsequently, the series version of the Cole impedance model parameters $\left(R_{\infty}, R_{1}, C_{\alpha}, \alpha\right)$ for the left and right biceps of all 18 participants were determined using a nonlinear least squares fitting (NLSF) procedure.

\section{RESULTS AND DISCUSSION}

\section{A. Data Reconstruction, Modified Fricke Models Design}

Mean values and corresponding standard deviations (SD) of series version of Cole model circuit parameters from preand post-fatigue impedance measured from the left as well as right biceps of participants in both groups, recalculated from dataset of 18 participants (Subsection II-A), are listed in Table I. Computed complete set of parameters of the modified Fricke model $\left(R_{\mathrm{ECF}}=R_{\infty}+R_{1}, R_{\mathrm{ICF}}=R_{\infty}\left(1+R_{\infty} / R_{1}\right)\right.$, $C_{\mathrm{M} \alpha}=C_{\alpha}\left(1+R_{\infty} / R_{1}\right)^{-2}$, while $\alpha$ remains the same) are also provided in the table. Comparison of total resistance $\left(R_{\infty}+R_{1}\right.$ vs. $\left.R_{\mathrm{ECF}}+R_{\mathrm{ICF}}\right)$ and pseudo-capacitance mean values in both versions of Cole impedance models of biceps tissues are depicted in Fig. 2. The parallel version of the Cole model is composed of significantly lower value of pseudocapacitance and higher values of resistances (light green region) compared to the series one (light orange region).
Due to the absence of commercially available CPEs, their implementation can be performed using active emulators [11] or via lumped RC chains [12]-[14]. Note that while the former requires advanced tools, the latter one is suitable for direct implementation in SPICE circuit simulators. Each CPE required for modeling the pre- and post-fatigue impedance measured from the left and right biceps of participants in both $60 \%$ and $75 \%$ 1-RM groups were replaced with Foster I RC network of three branches depicted in Fig. 1(b). Table II lists the Electronic Industries Alliance (EIA) standard compliant E96

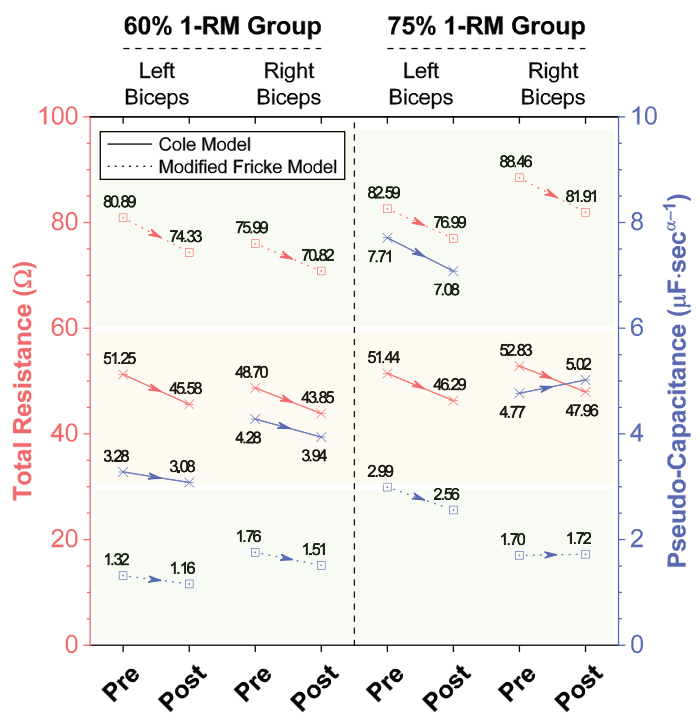

Fig. 2. Comparison of resistance and pseudo-capacitance mean values in bioimpedance models of biceps tissues listed in Table 1. 
TABLE II

Eia Standard Compliant E96 Series RC Values Used in Proposed CPEs via Foster I Network and Error Analysis.

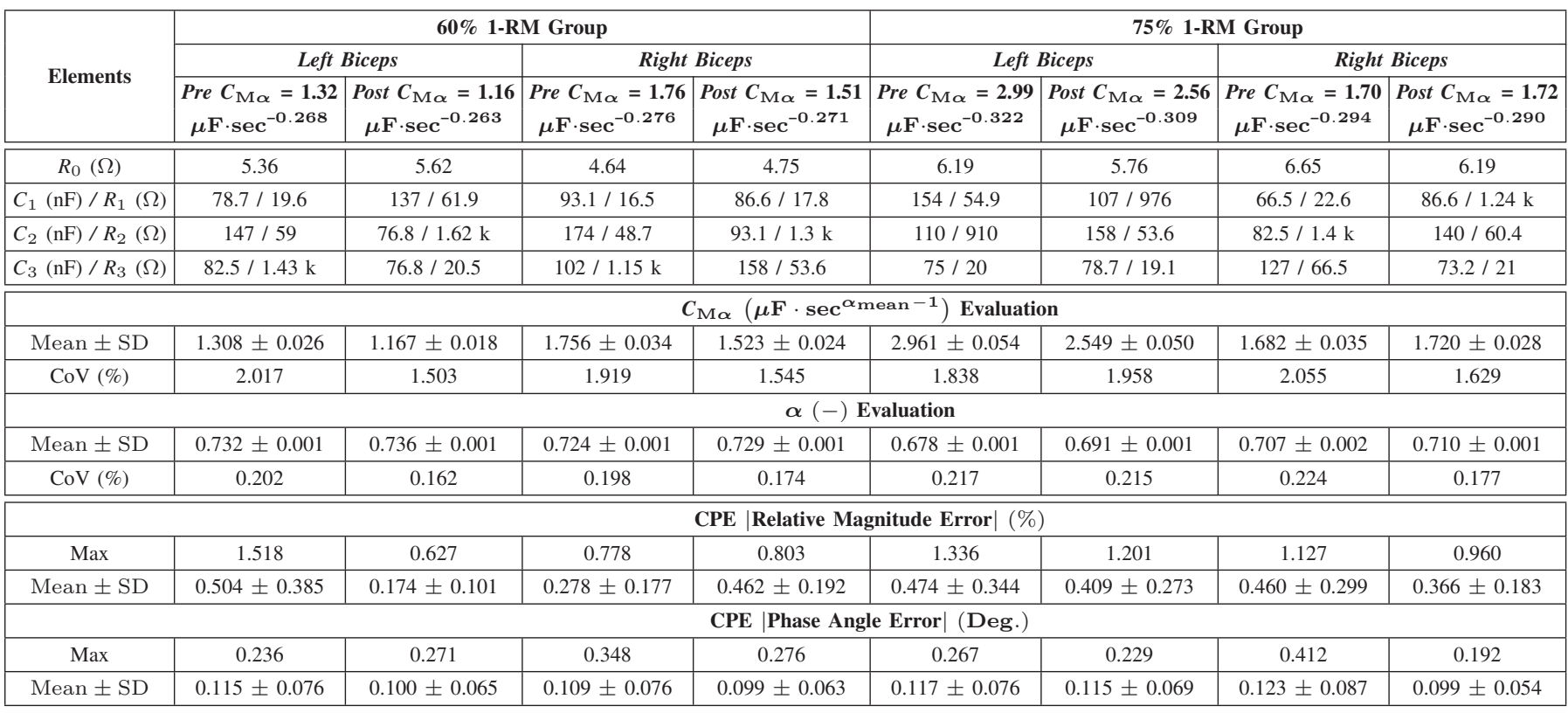

series $\mathrm{RC}$ values computed in frequency range from $10 \mathrm{kHz}$ to $100 \mathrm{kHz}$ using [13]. First of all, the behavior of CPEs were evaluated in terms of the coefficient of variation $(\mathrm{CoV})$ calculated as $\mathrm{CoV}=100 \cdot S D / \tilde{x}$, where $\tilde{x}$ is the mean value of CPEs' pseudo-capacitance or order. This indicator provides the relative standard deviation expressed in percentage. From the provided numerical analysis it is evident that the maximum $\mathrm{CoVs}$ of mean value of CPEs' pseudo-capacitance or order are $2.055 \%$ and $0.224 \%$, respectively. Secondly, the calculated maximum relative magnitude and phase angle errors of CPEs also show precise designs, i.e. $\pm 1.518 \%$ and $\pm 0.412^{\circ}$, respectively.

\section{Comparison of Bioimpedance Models}

Distribution of resistance and capacitance values required for realizing modified Fricke models of biceps tissues is shown in Fig. 3, while the comparison of total capacitance and resistance values is given in Table III. Note that this study supposes E96 series resistance values given as (R - E96) in Table I and RC values realizing CPEs listed in Table II.

For biological tissues the relationship between resistance $(R=|Z| \cdot \cos \theta)$ and reactance $(X=|Z| \cdot \sin \theta)$ of the complex impedance can be described with a circular arc in the Nyquist plot. 3D plots of series version of Cole and fitting modified Fricke models with parameters listed in Table I as well as modified Fricke model using E96 series RC values of the left and right biceps tissues of participants in the 60\% 1RM group are shown in Fig. 4. Corner frequencies and the frequency at which the reactance reaches its peak value $\left(f_{\mathrm{p}}=\omega_{\mathrm{p}} / 2 \pi=1 /\left\{2 \pi\left[C_{\mathrm{M} \alpha}\left(R_{\mathrm{ECF}}+R_{\mathrm{ICF}}\right)\right]^{-\alpha}\right\}\right)$ are also highlighted. Goodness of fitting of proposed modified Fricke
TABLE III

Comparison of Passive Component Values Required For REALIZING Modified Fricke Models of Biceps Tissues.

\begin{tabular}{|c|c|c|c|c|c|c|c|c|}
\hline \multirow{3}{*}{\begin{tabular}{c}
\multirow{3}{*}{$\begin{array}{c}\text { Evaluation } \\
\text { Criteria }\end{array}$} \\
\cline { 2 - 9 }
\end{tabular}} & \multicolumn{3}{|c|}{ 60\% 1-RM Group } & \multicolumn{3}{c|}{ 75\% 1-RM Group } \\
\cline { 2 - 9 } & Pre & Post & Pre & Post & Pre & Post & Pre & Post \\
\hline \hline Total Capacitance $(\mathrm{nF})$ & 308.2 & 290.6 & 369.1 & 337.7 & 339.0 & 343.7 & 276.0 & 299.8 \\
\hline Total Resistance $(\mathrm{k} \Omega)$ & 1.60 & 2.21 & 1.30 & 1.45 & 1.07 & 1.13 & 2.99 & 1.41 \\
\hline
\end{tabular}

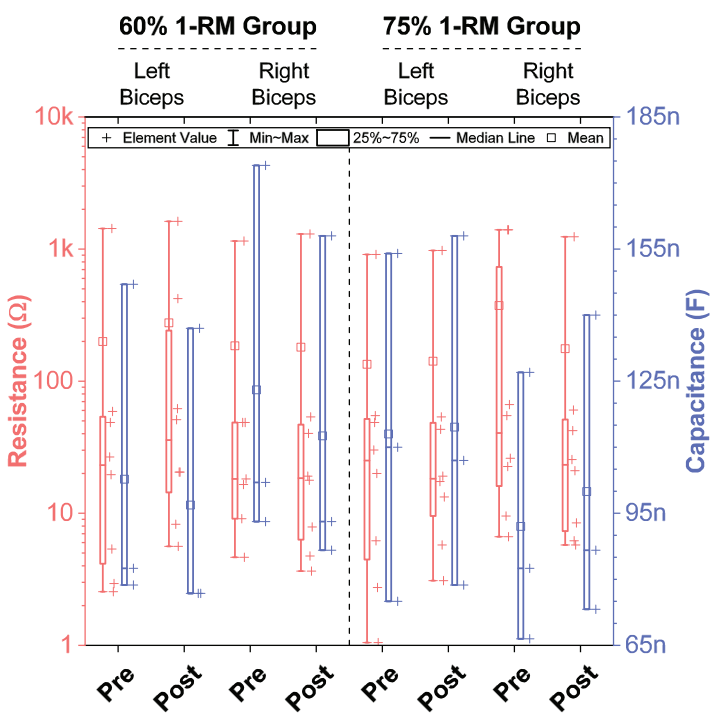

Fig. 3. Distribution of EIA standard compliant E96 series RC values used for realizing modified Fricke bioimpedance models of biceps tissues. 


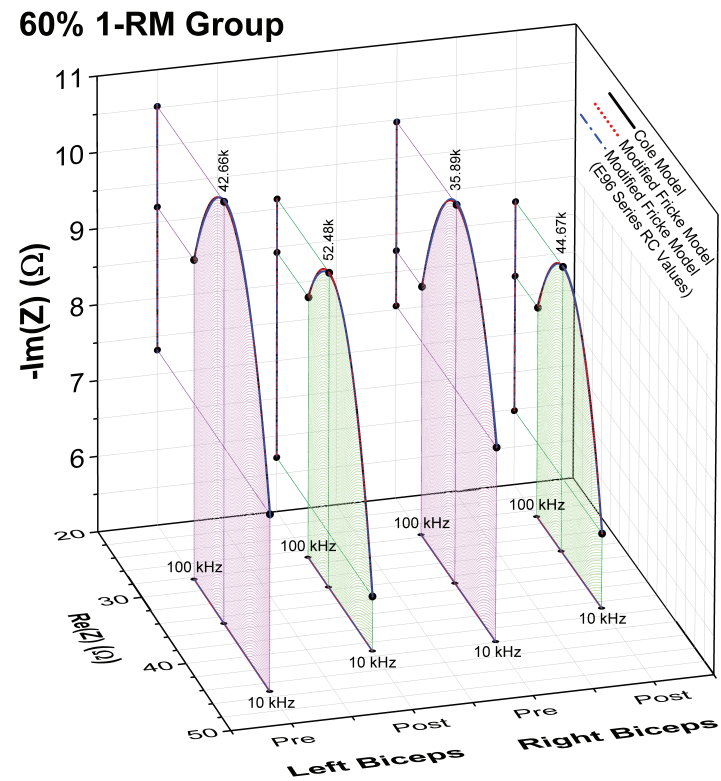

Fig. 4. 3D Nyquist plots of bioimpedance models of the left and right biceps tissues of participants in the 60\% 1-RM group. Selected frequencies are highlighted.

models vs. series version of Cole model data was evaluated by statistical metrics [15]. The root mean squared error (RMSE the square root of the average of squared errors), the coefficient of determination $\left(\mathrm{R}^{2}\right.$ - indicates the fraction of the fitting values that are closest to the line of reference data), and standard deviation were calculated for the resistance and reactance of the models complex electrical impedance. Note that pre- and post-fatigue impedance fitting modified Fricke model from the left and right biceps tissues in the both groups, either real or imaginary parts of the complex impedance, in terms of all studied indicators shows perfect fit to series version of Cole model data, i.e. $\mathrm{RMSE}_{\mathrm{R}, \mathrm{X}}=0 \Omega, \mathrm{R}_{\mathrm{R}, \mathrm{X}}^{2}=1.00(100 \%)$ and $\mathrm{SD}=0 \Omega$. Calculated RMSE and SD indicators for the modified Fricke model using E96 series RC values vs. series version of Cole model data are low (respectively vary between $0.016-0.059 \Omega$ and $0.026-0.065 \Omega$ ), while the fit accuracy in terms of coefficient of determination shows superb performance $\left(0.9978 \leq \mathrm{R}_{\mathrm{R}, \mathrm{X}}^{2} \leq 1.00\right)$. The statistical analysis results for the latter model are given in Table IV.

\section{CONCLUSiON}

This study showed that, although for modeling biological tissues the series version of Cole impedance model is widely used, the parallel version so-called modified Fricke impedance model should be preferred. Proposed practical models, while lacking in commercial unavailability of FOCs, leads to the belief that equivalents employing EIA standard compliant RC values are very helpful for practical studies of biological tissue structures. Statistical analysis results showed very good agreement with the initial dataset in terms of studied indicators.
TABLE IV

Statistical ANalysis of Fit ACCURACy of Proposed Modified Fricke Circuit Models Using RC Values FitTed to Data [3].

\begin{tabular}{|c|c|c|c|c|c|c|c|c|}
\hline \multirow{2}{*}{$\begin{array}{c}\text { Evaluation } \\
\text { Criteria }\end{array}$} & \multicolumn{4}{|c|}{ 60\% 1-RM Group } & \multicolumn{4}{c|}{ 75\% 1-RM Group } \\
\cline { 2 - 10 } & Left Biceps & \multicolumn{2}{|c|}{ Right Biceps } & Left Biceps & \multicolumn{2}{c|}{ Right Biceps } \\
\cline { 2 - 10 } & Pre & Post & Pre & Post & Pre & Post & Pre & Post \\
\hline \hline $\mathrm{RMSE}_{\mathrm{R}}(\Omega)$ & 0.044 & 0.019 & 0.039 & 0.044 & 0.057 & 0.038 & 0.059 & 0.033 \\
\hline $\mathrm{RMSE}_{\mathrm{X}}(\Omega)$ & 0.028 & 0.017 & 0.024 & 0.016 & 0.030 & 0.024 & 0.023 & 0.022 \\
\hline \hline $\mathrm{R}_{\mathrm{R}}^{2}(-)$ & 1.00 & 1.00 & 1.00 & 1.00 & 1.00 & 1.00 & 1.00 & 1.00 \\
\hline $\mathrm{R}_{\mathrm{X}}^{2}(-)$ & 0.9993 & 0.9998 & 0.9987 & 0.9996 & 0.9978 & 0.9987 & 0.9992 & 0.9993 \\
\hline \hline $\mathrm{SD}(\Omega)$ & 0.053 & 0.026 & 0.046 & 0.047 & 0.065 & 0.045 & 0.063 & 0.040 \\
\hline
\end{tabular}

\section{REFERENCES}

[1] S. B. Rutkove, "Electrical impedance myography: Background, current state, and future directions," Muscle \& Nerve, vol. 40, no. 6, pp. 936946, 2009. doi: 10.1002/mus.21362

[2] B. Sanchez and S. B. Rutkove, "Present Uses, Future Applications, and Technical Underpinnings of Electrical Impedance Myography," Current Neurology and Neuroscience Reports, vol. 17, no. 11, p. 86, 2017. doi: 10.1007/s11910-017-0793-3

[3] T. J. Freeborn and B. Fu, "Fatigue-Induced Cole Electrical Impedance Model Changes of Biceps Tissue Bioimpedance," Fractal and Fractional, vol. 2, no. 4, 2018. doi: 10.3390/fractalfract2040027

[4] L. K. Huang, L. N. Huang, Y. M. Gao, Ž. L. Vasić, M. Cifrek, and M. Du, "Electrical Impedance Myography Applied to Monitoring of Muscle Fatigue During Dynamic Contractions," IEEE Access, vol. 8, pp. 13 056-13 065, 2020. doi: 10.1109/ACCESS.2020.2965982

[5] T. J. Freeborn, G. Regard, and B. Fu, "Localized Bicep Tissue Bioimpedance Alterations Following Eccentric Exercise in Healthy Young Adults," IEEE Access, vol. 8, pp. 23 100-23 109, 2020. doi: 10.1109/ACCESS.2020.2970314

[6] H. Fricke, "The electric conductivity and capacity of disperse systems," Journal of Applied Physics, vol. 1, no. 2, pp. 106-115, 1931. doi: 10.1063/1.1744988

[7] K. Biswas, G. Bohannan, R. Caponetto, A. M. Lopes, and J. A. T. Machado, Fractional-order devices. Springer, 2017. ISBN 978-3-319$54459-5$

[8] K. S. Cole, "Permeability and impermeability of cell membranes for ions," Cold Spring Harbor Symposia on Quantitative Biology, vol. 8, pp. 110-122, 1940. doi: 10.1101/SQB.1940.008.01.013

[9] S. Grimnes and Ø. G. Martinsen, Bioimpedance and Bioelectricity Basics. Oxford: Academic Press, 2015. ISBN 978-0-12-411470-8

[10] M. Guermazi, O. Kanoun, and N. Derbel, "Investigation of Long Time Beef and Veal Meat Behavior by Bioimpedance Spectroscopy for Meat Monitoring," IEEE Sensors Journal, vol. 14, no. 10, pp. 3624-3630, 2014. doi: 10.1109/JSEN.2014.2328858

[11] G. Tsirimokou, A. Kartci, J. Koton, N. Herencsar, and C. Psychalinos, "Comparative Study of Discrete Component Realizations of FractionalOrder Capacitor and Inductor Active Emulators," Journal of Circuits, Systems and Computers, vol. 27, no. 11, p. 1850170, 2018. doi: $10.1142 / \mathrm{S} 0218126618501700$

[12] J. Scott and P. Single, "Compact Nonlinear Model of an Implantable Electrode Array for Spinal Cord Stimulation (SCS)," IEEE Transactions on Biomedical Circuits and Systems, vol. 8, no. 3, pp. 382-390, 2014. doi: 10.1109/TBCAS.2013.2270179

[13] A. Kartci, A. Agambayev, M. Farhat, N. Herencsar, L. Brancik, H. Bagci, and K. N. Salama, "Synthesis and Optimization of Fractional-Order Elements Using a Genetic Algorithm," IEEE Access, vol. 7, pp. 80233 80 246, 2019. doi: 10.1109/ACCESS.2019.2923166

[14] A. Kartci, N. Herencsar, J. T. Machado, and L. Brancik, "History and Progress of Fractional-Order Element Passive Emulators: A Review," Radioengineering, vol. 29, no. 2, pp. 296-304, June 2020. doi: 10.13164/re.2020.0296

[15] C. A. Gueymard, "A review of validation methodologies and statistical performance indicators for modeled solar radiation data: Towards a better bankability of solar projects," Renewable and Sustainable Energy Reviews, vol. 39, pp. 1024-1034, 2014. doi: 10.1016/j.rser.2014.07.117 